\title{
Urodynamic evaluation of patients with autosomal dominant pure spastic paraplegia linked to chromosome $2 \mathrm{p} 21-\mathrm{p} 24$
}

\author{
L Neerup Jensen, T Gerstenberg, E B Kallestrup, P Koefoed, J Nordling, J E Nielsen
}

Department of Urology, Herlev Hospital, University of Copenhagen, Denmark L Neerup Jensen T Gerstenberg E B Kallestrup J Nordling

Institute of Medical Biochemistry and Genetics, Department of Medical Genetics, Section of

Neurogenetics,

University of

Copenhagen, Denmark

P Koefoed

J E Nielsen

Correspondence to: Dr J E Nielsen, Institute of Medical Biochemistry and Genetics, Department of Medical Genetics, Section of Neurogenetics, The Panum Institute, Building 24.4, Blegdamsvej 3, DK-2200, Copenhagen N, Denmark. Telephone 00453532 7816; fax 00453532 7845; email jnielsen@medgen.imbg.ku.dk

Received 15 January 1998 and in revised form

5 March 1998

Accepted 19 March 1998

\begin{abstract}
Objectives-There are at least three clinically indistinguishable but genetically different types of autosomal dominant pure spastic paraplegia (ADPSP). Lower urinary tract symptoms are often present but have not been described in a homogeneous patient population. In this study lower urinary tract symptoms, cystometrical, and neurophysiological characteristics are described in patients with ADPSP linked to chromosome 2p21-p24.

Methods-Lower urinary tract symptoms were recorded at an interview and according to a formalised questionnaire. Eleven patients were clinically evaluated and cystometry, measurements of the cutaneous perception threshold, bulbocavernosus reflex latency, and somatosensory evoked potentials (SSEPs) of the pudendal nerve were performed.

Results-All patients experienced urinary urgency or urge incontinence. Rectal urgency and sexual dysfunction were reported by most patients. The cystometrical findings showed a mixed pattern of bladder dysfunction. The SSEPs were normal in all but the bulbocavernosus reflex latency was significantly prolonged in seven patients and the cutaneous perception threshold was raised in five patients.

Conclusions-Lower urinary tract symptoms and probably also bowel and sexual dysfunction in patients with ADPSP linked to chromosome 2p21-p24 are due to a combination of somatic and autonomic nervous system involvement which support the proposed multisystem affection in ADPSP linked to chromosome 2p21-p24.

(f Neurol Neurosurg Psychiatry 1998;65:693-696)
\end{abstract}

Keywords: autosomal dominant pure spastic paraplegia linked to chromosome $2 \mathrm{p}$; voiding; bowel; sexual dysfunction; urodynamics; neurophysiology

Hereditary spastic paraplegia is a heterogeneous group of rare neurodegenerative disorders of the motor system characterised by slowly progressive spasticity and weakness of the lower limbs.

Based on the clinical features they are divided into two groups, depending on whether the disorder is a pure spastic paraplegia or a more complex syndrome with other codominating signs ${ }^{1}$ such as amyotrophy, mental retardation, eye symptoms, epilepsy, ataxia, dystonia, and peripheral neuropathy. ${ }^{2}$ Additional but not obligatory features seen in the pure form which predominantly affects legs may be hyperreflexia and weakness of the upper limbs, disturbance of sphincter function, and mild sensory impairment. In pure hereditary spastic paraplegia, inheritance is most commonly autosomal dominant. ${ }^{3}$ By linkage analyses autosomal dominant pure spastic paraplegia (ADPSP) has been mapped to the chromosomes $14 \mathrm{q}, 2 \mathrm{p}$, and $15 \mathrm{q}^{4-6}$ and the "spastic paraplegia" is likely to be a symptom due to variable mutations of various genes in the CNS involving more components of the corticospinal tracts (neuron, axon, myelin, interneuron, synapse, and receptors). ${ }^{7}$ A dynamic CAG repeat expansion has recently been identified to be the most likely disease causing mutation in ADPSP linked to chromosome $2 \mathrm{p} 21-\mathrm{p} 24 .^{8}$ The neuropathological feature of ADPSP is axonal degeneration that is maximal in the terminal portions of the longest descending and ascending tracts (crossed and uncrossed corticospinal tracts to the legs, fasciculus gracilis fibres, and to a lesser extent, spinocerebellar fibres) whereas the neuronal cell bodies of the degenerating fibres are preserved. Dorsal root ganglia, posterior roots, and peripheral nerves are normal. ${ }^{9} 10$

Lower urinary tract symptoms are often present, but most reports on voiding symptoms in hereditary spastic paraplegia are descriptive and only few patients of unknown inheritance and family history have been urodynamically evaluated. Urodynamic evaluation of patients from families with ADPSP with known genetic localisation assuring a homogeneous patient group has not previously been reported. Here we describe the urodynamic findings in 11 patients from five different families with ADPSP linked to chromosome 2p21-p24.

\section{Patients and methods}

In six of eight families with ADPSP linked to chromosome 2p21-p24, lower urinary tract symptoms were present in 16 of 44 definitely affected members. Family details and clinical features as well as neurophysiological and MRI findings in five of these families are previously described. ${ }^{11}$ After informed consent was obtained, two patients from family A, four from family C, three from family E, and one patient from each of the families $\mathrm{K}$ and $\mathrm{L}$ were studied. Five patients refused participation.

Genitourinary symptoms were recorded both at an interview and according to a formalised questionnaire. Each patient filled in a frequency/volume chart at home during 48 hours to assess the voiding symptoms. Urody- 
Table 1 Clinical data of the patients with ADPSP and LUTS

\begin{tabular}{|c|c|c|c|c|c|c|c|c|c|c|c|}
\hline & \multicolumn{11}{|c|}{ Family, patient No } \\
\hline & $A 1$ & $A 2$ & C3 & $C 4$ & C5 & C6 & E7 & E8 & E9 & K10 & $L 11$ \\
\hline Sex & $\mathrm{F}$ & $\mathrm{F}$ & $M$ & $M$ & $\mathrm{~F}$ & M & $M$ & $\mathrm{~F}$ & $M$ & $M$ & $M$ \\
\hline Age $(y)$ & 56 & 37 & 58 & 33 & 55 & 41 & 61 & 55 & 34 & 50 & 43 \\
\hline \multicolumn{12}{|l|}{ Age at onset (y): } \\
\hline Neurological symptoms & 30 & 1 & 42 & 23 & 43 & 33 & 35 & 20 & 5 & 38 & 18 \\
\hline Urological symptoms & 30 & 18 & 42 & 23 & 50 & 39 & 43 & 35 & 24 & 47 & 38 \\
\hline \multicolumn{12}{|l|}{ Symptoms: } \\
\hline Urgency & +++ & ++ & ++ & ++ & ++ & + & \pm & +++ & + & ++ & ++ \\
\hline Urge incontinence & ++ & - & + & + & + & - & \pm & ++ & - & + & - \\
\hline Frequency & ++ & + & ++ & - & ++ & + & \pm & ++ & - & ++ & + \\
\hline Nocturia & + & +++ & + & - & + & - & \pm & - & - & - & - \\
\hline Hesitancy & - & +++ & + & + & - & - & \pm & - & + & ++ & + \\
\hline Diminished stream & - & + & + & - & + & - & \pm & - & - & ++ & + \\
\hline Rectal urge & - & + & + & +++ & +++ & + & + & + & + & ++ & + \\
\hline Rectal urge incontinence & - & + & - & ++ & + & + & + & + & - & + & + \\
\hline
\end{tabular}

+=mild; ++=moderate; +++=severe;-=absent; $\pm=$ indeterminant

namic evaluation was performed with patients in the supine position using a double lumen transurethral catheter, rectal pressure balloon, and two EMG surface electrodes placed in the region of the external anal sphincter. If possible a free flow study was performed on arrival. Patients were then catheterised and postvoid residual urine volume measured. Cystometry was performed using body warm saline infused at $50 \mathrm{ml} / \mathrm{min}$. Intravesical and rectal pressures were recorded simultaneously with the EMG activity on a multichannel recorder (MMS UD-2000). Definitions conform to the International Continence Society (ICS) standards. ${ }^{12}$ The pelvic floor reflexes were graded arbitrary from absent $(0)$, weak $(+)$, moderate $(++)$, to normal $(+++)$. Rectal examination was done to evaluate prostate size in the male patients and function of the anal sphincter in all patients.

Measurements of the cutaneous perception threshold, bulbocavernosus reflex latency, and somatosensory evoked potentials (SSEPs) of the pudendal nerve were performed.

A surface stimulator was used for stimulation of the skin over the dorsal nerve of the penis or clitoris.

The sensory threshold was defined as the lowest current perceptible. A mean of three measurements was used for calculation.

In bulbocavernosus reflex testing a needle electrode was placed in the external anal sphincter muscle. The skin over the dorsal nerve of the penis or clitoris was stimulated supramaximally with a current at least three times as strong as the perception threshold. The interval from stimulation to onset of the response was recorded as the latency.

To obtain cortical evoked potentials an electroencephalography platinum electrode was placed at the scalp over the sensory cortex at a point $2 \mathrm{~cm}$ behind the middle of a line from the glabella to the inion $(\mathrm{Cz}-2)$ and a reference electrode at the forehead (Fpz). Twofold serial stimulations just above the perception threshold were averaged and interposed (Neuromatic 2000C, Dantec, Skovlunde, Denmark). The latencies from stimulation to the first, second, and third positive and negative deflections were calculated and the amplitude was measured. ${ }^{13}$

\section{Results}

The family, sex, and age distribution of the 11 patients studied are shown in table 1 . The mean duration of ADPSP was 21 years and the mean duration of lower urinary tract symptoms was 12 years. Most patients developed bladder dysfunction several years after the first neurological symptoms but in three patients lower urinary tract symptoms were part of the presenting symptom complex. Five patients had undergone urodynamic evaluation at an earlier date in other hospitals, none of them receiving any oral medications at the time of examination. Two patients (Nos 2 and 5) experienced some benefit from clean intermittent self catheterisation, but patient No 2 stopped after some time because of complications. Patient No 7 recently had a urodynamic evaluation disclosing severe detrusor hyperreflexia and no detrusor-sphincter dyssynergia; having failed a trial of several anticholinergic drugs, he preferred suprapubic catheter drainage.

An accurate description of the symptoms was not possible in the patient with the indwelling catheter.

A detailed description of lower urinary tract symptoms in the remaining 10 patients is shown in table 1 .

Urinary urgency and frequency were the dominant complaints and six patients regularly experienced urge incontinence. Other lower urinary tract symptoms included nocturia, hesitancy, and diminished force of stream. Rectal urgency was reported by all patients but one, and eight patients occasionally experienced rectal urge incontinence.

Sexual dysfunction was reported by seven patients (two women and five men). The male complaints varied from slightly reduced erection to totally loss of the ability to obtain erection. The women reported reduction or lack of lubrication and reduction in orgasmic capacity. One woman did not answer the questions concerning sexual function.

The cystometrical findings are shown in table 2. We found a mixed pattern of bladder dysfunction; six patients demonstrated normal detrusor activity (patient Nos 1-4, 6, and 11), patient No 5 was found to have detrusor hyporeflexia with delayed first sensation and three patients showed detrusor hyperreflexia (patient Nos 8, 9, and 10). In seven patients satisfactory sphincter EMG recordings were obtained, all of them being normal. Postvoid residual urine volumes were raised in all 
Table 2 Cystometrical findings in the patients with ADPSP and LUTS

\begin{tabular}{|c|c|c|c|c|c|c|c|c|c|c|c|}
\hline & \multicolumn{11}{|c|}{ Patient No } \\
\hline & 1 & 2 & 3 & 4 & 5 & 6 & 7 & 8 & 9 & 10 & 11 \\
\hline PVR (ml) & 90 & 110 & 80 & 60 & 150 & 100 & \pm & 60 & 0 & 180 & 15 \\
\hline First sensation $(\mathrm{ml})$ & 26 & 36 & 41 & 156 & 349 & 78 & \pm & 35 & 18 & 52 & 111 \\
\hline Capacity $(\mathrm{ml})$ & 306 & 486 & 161 & 353 & 471 & 325 & \pm & 76 & 390 & 209 & 400 \\
\hline Hyperreflexia & - & - & - & - & - & - & \pm & + & + & + & - \\
\hline DSD & - & - & - & - & \pm & \pm & \pm & - & - & - & \pm \\
\hline
\end{tabular}

+=present;-=absent; $\pm=$ indeterminant; PVR=post-void residual urine volume. $\mathrm{DSD}=$ detrusor-sphincter dyssynnergia.

patients but two. Seven patients had an early first sensation; the capacity being almost normal in all but one patient (No 8). In the male patients rectal examination showed normal prostate size and consistency for age. Clinically, the bulbocavernosus reflex was absent in six patients and only normal in one (patient No 10), as the anal and anocutaneous reflexes were reduced in all but patient No 10 (table 3). The anal sphincter tone was decreased in the same 10 patients. The cutaneous perception threshold was raised in five patients. SSEPs were normal in all patients. The bulbocavernosus reflex latency was significantly prolonged in seven patients with an obvious correlation between a clinically absent bulbocavernosus reflex and prolongation of the bulbocavernosus reflex latency. There were no correlations between the duration of symptoms, the three point functional grading score, urological symptoms, and cystometrical and neurophysiological findings.

\section{Discussion}

The frequency of urinary symptoms in our patients $(36 \%)$ correlates well with the frequency of $34 \%$ reported by Dürr et $a l^{14}$ in 83 patients with definite ADPSP linked to chromosome 2 . However, they reported a sex difference, the women more often having sphincter disturbances than men. In our patients there was a higher proportion of men reporting lower urinary tract symptoms but this difference is probably due to the relatively few patients and most certainly does not rely on a real biological variation. Urinary symptoms most often are late manifestations $\mathrm{s}^{15}$ but in three patients, those symptoms were present from the onset of the disease. In agreement with other reports ${ }^{14-16}$ the most common symptoms were urinary urgency, urge incontinence, and frequency. Urinary urgency has been extensively reported, but rectal urgency is rarely described. Bushman et $a l^{16}$ found two of three patients with rectal urgency and Cartlidge and Bone ${ }^{17}$ described one of three brothers with rectal urgency. A high proportion of bowel symptoms has been reported by Scheltens $e t a l^{18}$ who found a predominance of faecal incontinence over urinary incontinence in a large Dutch family. In other diseases-for example, multiple sclerosis - there is a correlation between sexual dysfunction, weakness of the pelvic floor, bladder, and bowel dysfunction and spasticity. ${ }^{19} 20$ Therefore the finding of sexual dysfunction associated with ADPSP is not surprising; on the contrary, the absence of alterations in sexual function in most studies on hereditary spastic paraplegia is conspicuous.

In seven patients urinary urgency/urge incontinence was not due to detrusor hyperreflexia; however, five of these patients had an early first sensation thus indicating a sensory urgency/urge incontinence. This finding may suggest an autonomic component which, in combination with the weak pelvic floor, might cause the rectal urgency/urge incontinence; however, in the very few studies trying to uncover other changes in autonomic function-for example, sweat regulation and vasomotor function-the results were normal. ${ }^{1617}$

In the eight patients showing raised postvoid residual urine volumes, an obstructive aetiology or detrusor weakness might be proposed. Bushman et $a l^{16}$ reported detrusor-sphincter dyssynergia in one of three patients with hereditary spastic paraplegia of unknown inheritance and family history. The EMG recording was not satisfactory in four of our patients; however, hyperreflexia was not disclosed in those cases, therefore the raised postvoid residual urine volumes cannot be explained by detrusor-sphincter dyssynergia. In the three patients in whom hyperreflexia was noted a spinal pathology could be responsible for the unstable detrusor without evidence of an organic or neurogenic increase in outlet resistance.

The weakness of the pelvic floor correlates well with the finding of hyporeflexia, indicating

Table 3 Clinical evaluation of reflexes, anal sphincter function and the neurophysiological findings in the patients with ADPSP and LUTS

\begin{tabular}{|c|c|c|c|c|c|c|c|c|c|c|c|}
\hline & \multicolumn{11}{|c|}{ Patient No } \\
\hline & 1 & 2 & 3 & 4 & 5 & 6 & 7 & 8 & 9 & 10 & 11 \\
\hline BC reflex & 0 & 0 & 0 & 0 & + & + & ++ & 0 & ++ & +++ & 0 \\
\hline Anal reflex & + & + & + & + & ++ & + & ++ & 0 & ++ & +++ & + \\
\hline Anocutaneous reflex & + & + & + & + & ++ & + & ++ & + & ++ & +++ & + \\
\hline Anal sphincter function & + & + & + & + & + & + & ++ & + & ++ & +++ & + \\
\hline Cutaneous perception & 2.6 & 1.9 & 4.0 & 2.8 & 8.1 & 3.9 & 9.9 & 5.5 & 4.9 & 3.8 & 5.7 \\
\hline $\mathrm{BC}$ reflex latency & 63 & 129 & 61 & 123 & 56 & 37 & 44 & 45 & 30 & 36 & 31 \\
\hline
\end{tabular}

$\mathrm{BC}=$ bulbocavernosus: $0=$ absent; $+=$ weak $;++=$ moderate; $+++=$ normal. Cutaneous perception: normal values: men: median (range) 3.3 (3.0-4.2) mAmp/0.2 ms/2 Hz, women: 2.7 (2.0-3.8) mAmp/0.2 ms/2 Hz. BC latency: normal values: men and women: median (range): $36(30-40) \mathrm{ms}$. 
that a peripheral neuropathy may be part of the pathophysiological picture. This is in accordance with the finding of prolongation of the bulbocavernosus reflex latencies, suggesting dysfunction of the integrity of central and peripheral tracts of the sacral reflex arc that consist of the dorsal nerve of the clitoris or penis, the S2-S4 cord segments, and the motor branch of the pudendal nerve innervating the bulbocavernosus muscles, and anal and urethral sphincter. ${ }^{21}$ The bulbocavernosus reflex has been evaluated clinically compared with the EMG demonstration of the reflex; the absence of a bulbocavernosus reflex in male patients was found to be indicative of a neurological lesion involving the sacral cord and highly suggestive of such a lesion in female patients. $^{22}$ The normal SSEPs are in accordance with the normal findings from upper and lower limbs previously reported in 16 patients with ADPSP linked to chromosome $2 \mathrm{p} 21-\mathrm{p} 24 .{ }^{11}$ As the SSEPs were normal in all cases the prolonged bulbocavernosus refluex latencies indicate affection of the motor part of the somatic pudendal nerve. The delay of the bulbocavernosus reflex, however, may also be located in the sacral cord, involving interneurons connecting the afferent and efferent part of the bulbocavernosus reflex arc. The cutaneous perception threshold has been evaluated in males only. It is thought to give a semiquantitative measure of sensory nerve fibres mediating pain, different from those of the dorsal columns responsible for the SSEPs; and the raised cutaneous perception thresholds therefore suggest an affection of those fibres of the sensory part of the pudendal nerve. ${ }^{23}$

A high incidence of multisystem subclinical involvement of the CNS has been proposed by different neurophysiological techniques and MRI of the brain also ${ }^{24}{ }^{25}$ and the degenerative processes seem not only to be confined to the long motor and sensory tracts. We propose that lower urinary tract symptoms and probably also bowel and sexual dysfunction in patients with ADPSP linked to chromosome 2p21-p24 are due to a combination of somatic and autonomic nervous system involvement supporting the proposed multisystem affection in ADPSP. However, this study is the first on ADPSP linked to chromosome 2p21-p24 and therefore more studies are warranted to elucidate further aspects of the pathophysiological background of the bladder, bowel, and sexual dysfunction so obviously associated with the disease.

Financial support from the Danish Medical Research Council; the Danish Medical Association Research Fund; the Dagmar
Marshall Fund and the Dr Eilif Trier-Hansen and wife Ane Trier Hansen Fund is gratefully acknowledged. We thank Elsemarie Maegaard for her technical assistance, Dr Sven Asger Sorensen for critically reviewing of the manuscript, and the patients without whom this study would not have been possible.

1 Harding AK. Classification of the hereditary ataxias and paraplegias. Lancet 1983;i:1151-5.

2 Bruyn RPM, Scheltens PH. Hereditary spastic paraparesis (Strümpell-Lorrain). In: Vinken PJ, Bruyn GW, eds. Handbook of clinical neurology, 2 nd series. Amsterdam: North Holland Publishing, 1991;15:301-8.

3 Holmes GL, Shaywitz BA. Strümpell's pure familial spastic paraplegia: case study and review of the literature. 7 Neurol paraplegia: case study and review of the
Neurosurg Psychiatry 1977;40:1003-8.

4 Hazan J. Lamy C, Melki J, et al. Autosomal dominant familial spastic paraplegia is genetically heterogeneous and one locus maps to chromosome 14q. Nat Genet 1993;5:163-7.

5 Hazan J, Fontaine B, Bruyn RPM, et al. Linkage of a new locus for autosomal dominant familial spastic paraplegia to chromosome 2p. Hum Mol Genet 1994;3:1569-73.

6 Fink JK, Wu CB, Jones SM, et al. Autosomal dominant familial spastic paraplegia: tight linkage to chromosome 15q. Am Ұ Hum Genet 1995;56:188-92.

7 Kobayashi H, Garcia CA, Alfonso G, et al. Molecular genetics of familial spastic paraplegia: a multitude of responsible genes. F Neurol Sci 1996:137:131-8.

8 Nielsen JE, Koefoed P, Abell K, et al. CAG repeat expansion in autosomal dominant pure spastic paraplegia linked to chromosome 2p21-p24. Hum Mol Genet 1997;6:1811-6.

9 Behan WMH, Maia M. Strümpell's familial spastic paraplegia: genetics and neuropathology. $\mathcal{F}$ Neurol Neurosurg Psychiatry 1974;37:8-20.

10 Bruyn RPM. The neuropathology of hereditary spastic paraparesis. Clin Neurol Neurosurg 1992;94(suppl):S16-8.

11 Nielsen JE, Krabbe K, Jennum P, et al. Autosomal dominant pure spastic paraplegia: a clinical, paraclinical and genetic study. F Neurol Neurosurg Psychiatry 1998;64:61-6.

12 Abrams P, Blaivas JG, Stanton SL, et al. Standardisation of terminology of lower urinary tract function. Neurourol Urodyn 1988;7:403-27.

13 Gerstenberg TC, Nordling J, Hald T. Evoked potentials from the lower urinary tract 11. The spinocortical neuraxis. Scand f Urol Nephrol 1991;138(suppl):41-6.

14 Dürr A, Davoine CS, Paternotte C, et al. Phenotype of autosomal dominant spastic paraplegia linked to chromosome 2. Brain 1996;119:1487-96.

15 Harding AK. Hereditary "pure" spastic paraplegia: a clinical and genetic study of 22 families. I Neurol Neurosurg Psychiatry 1981;44:871-83.

16 Bushman W, Steers WD, Meythaler JM. Voiding dysfunction in patients with spastic paraplegia: urodynamic evaluation and response to continuous intrathecal baclofen. ation and response to continuous

17 Cartlidge NEF, Bone G. Sphincter involvement in hereditary spastic paraplegia. Neurology 1973;23:1160-3.

18 Scheltens P, Bruyn RMP, Hazenberg GJ. A Dutch family with autosomal dominant pure spastic paraparesis (Strümpell's disease). Acta Neurol Scand 1990;82:169-73.

19 Stenager E, Nylev Stenager E, Jensen K. Sexual aspects of multiple sclerosis. Semin Neurol 1992;12:120-4.

20 Hulter BM, Lundberg PO. Sexual function in women with advanced multiple sclerosis. $\mathcal{F}$ Neurol Neraosurg Psychiatry 1995;59:83-6.

21 Opsomer RJ, Caramia MD, Zarola F, et al. Neurophysiological evaluation of central-peripheral sensory and motor pudendal fibres. Electroencephalography and Clinical Neurophysiology 1989;74:260-70.

22 Blaivas JG, Zayed AAH, Labib KB. The bulbocavernosus reflex in urology: a prospective study of 299 patients. $F$ Urol 1981;126:197-9.

23 Gerstenberg TC, Nordling J, Hald T, et al. Standardized evaluation of erectile dysfunction in 95 consecutive evaluation of erectile dysfunction
patients. F Urol 1989;141:857-62.

24 Tedeschi G, Allocca S, Di Costanzo A, et al. Multisystem involvement of the central nervous system in Strümpell's disease. A neurophysiological and neuropsychological study. $\mathcal{F}$ Neurol Sci 1991;103:55-60.

25 Krabbe K, Nielsen JE, Fallentin E, et al. MRI of autosomal dominant pure spastic paraplegia. Neuroradiol 1997;39: $724-7$. 